\title{
Ordered vacancy network induced by the growth of epitaxial graphene on $\operatorname{Pt}(111)$.
}

\author{
G. Otero,${ }^{1}$ C. Gonzalez,${ }^{1}$ A.L. Pinardi,${ }^{1}$ P. Merino, ${ }^{2}$ S. Gardonio,${ }^{3}$ S. Lizzit,${ }^{3}$ M. Blanco-Rey,${ }^{4}$ \\ K. Van de Ruit, ${ }^{5}$ K. Flipse, ${ }^{5}$ J. Mendez, ${ }^{1}$ P.L. de Andres, ${ }^{1}$ and J.A. Martin-Gago ${ }^{1,2}$ \\ ${ }^{1}$ Instituto de Ciencia de Materiales de Madrid (CSIC) E-28049 Cantoblanco, Madrid, SPAIN \\ ${ }^{2}$ Centro de Astrobiologia, INTA-CSIC, Torrejon de Ardoz, E-28850 Madrid, SPAIN \\ ${ }^{3}$ Sincrotrone Trieste S.C.p.A., Strada Statale 14, km. 163.5, I-34149 Trieste, ITALY \\ ${ }^{4}$ Department of Chemistry, University of Cambridge, Cambridge CB2 1EW, UK \\ ${ }^{5}$ Dept. of Applied Physics, Eindhoven University of Technology, \\ P.O. Box 513, 5600 MB Eindhoven, The Netherlands
}

(Dated: June 7, 2011)

\begin{abstract}
We have studied large areas of $\sqrt{3} \times \sqrt{3} \mathrm{R} 30^{\circ}$ graphene commensurate with a $\mathrm{Pt}(111)$ substrate. A combination of experimental techniques with ab-initio density functional theory leads us to conclude that this structure is originated by the formation of a surface reconstruction at the $\mathrm{Pt}$ surface, consisting of an ordered vacancy network in the outermost Pt layer and a graphene layer covalently bounded to the Pt substrate. This reconstruction is enhanced if low temperatures and polycyclic aromatic hydrocarbons are used as molecular precursors for the epitaxial growth of graphene.
\end{abstract}

PACS numbers: $68.43 . \mathrm{Bc}, 68.55 .-\mathrm{a}, 82.65 .+\mathrm{r}$

Recent experiments on epitaxial graphene layers grown on metal surfaces have raised hopes of developing a large number of applications.[1, 2] Therefore, understanding the growth, morphology and structure of these layers is an active research subject owing to its fundamental and practical interest. Graphene layers can be efficiently grown by thermal decomposition of small organic molecules, usually ethylene, on transition metals, such as $\operatorname{Ru}(0001), \operatorname{Ir}(111), \operatorname{Rh}(111)$, and $\operatorname{Pt}(111) \cdot[3-8]$ The morphology and structure of the graphene layer are intensively discussed in the literature, and the strength and nature of the interaction of the layer with the metal substrate is still under debate. The interaction of nanometric size graphene in the form of islands, patches or defective areas with the supporting metallic substrate could give rise to tailored electronic properties, as it has been recently reported.[10,11] This interaction is directly related to the distance between the metal surface and the graphene layer, and determines, for instance, the amount of the rippling on the Moire patterns. Earlier theoretical models have used a simple parameterized tight-binding approach relying on a weak interaction between both systems (large separation), while models based in ab-initio approaches have argued in favor of shorter distances and a stronger interaction (e.g. $[3,4])$.

Typical for graphene on transition metal surfaces is the development of Moire patterns exhibiting large surface unit supercells with periodicities of about $2-3 \mathrm{~nm}$. Ru and Ir surfaces develop extended single-domain graphene layers, as depicted by Scanning Tunneling Microscopy (STM) images. [3, 5] Pt, on the contrary, shows incommensurate domains coexisting with Moire's of different periodicities on the same surface.[7,8] In this work we focus our interest on the formation and structure of previously reported graphene regions, which are commensurate with a $\mathrm{Pt}(111)$ substrate in small crystal-

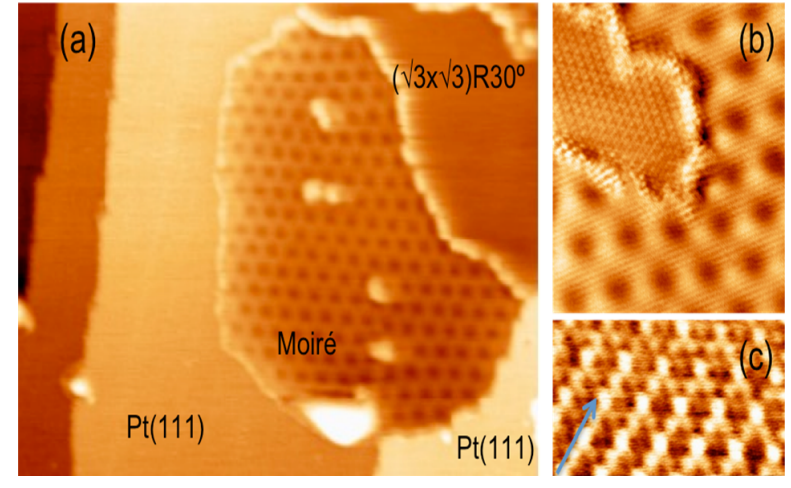

FIG. 1. (Color online) Constant-current STM images displaying islands exhibiting the $\sqrt{3} \times \sqrt{3} \mathrm{R} 30^{\circ}$ structure. Scanned areas are (a) $53 \times 36 \mathrm{~nm}^{2}$, (b) $12 \times 12 \mathrm{~nm}^{2}$ and (c) $3.2 \times 1.3$ $\mathrm{nm}^{2}$. Bias voltages range from 20 to $100 \mathrm{mV}$ and tunnel currents from 0.1 to $2 \mathrm{nA}$. The arrow indicates the $[11 \overline{2}]$ surface direction. Image (a) was recorded for a partially covered surface, where the growth process was stopped before saturation. Panels (a) and (b) shows the $\sqrt{3} \times \sqrt{3} \mathrm{R} 30^{\circ}$ superstructure coexisting with Moires region.

lographic ratios, i.e. forming a $\sqrt{3} \times \sqrt{3} \mathrm{R} 30^{\circ}\left(\mathrm{R} 30^{\circ}\right.$ hereafter) periodicity.[7] We show that the R30 ${ }^{\circ}$ structure is the result of a large surface- overlayer interaction that accommodates its large mismatch by inducing a strong reconstruction on the Pt surface. These regions, formed with smaller crystallographic ratios than the Moire's counterpart, make the system amenable to be explored by ab-initio calculations, an important tool to accurately interpret the chemistry and physics of epitaxial graphene. $[4,11,12]$ Therefore, this system offers a unique opportunity to test theories and structural parameters that are relevant to understand the interaction of graphene with transition metal surfaces.

To prepare wide $\mathrm{R} 30^{\circ}$ areas we slowly evaporate (up to 


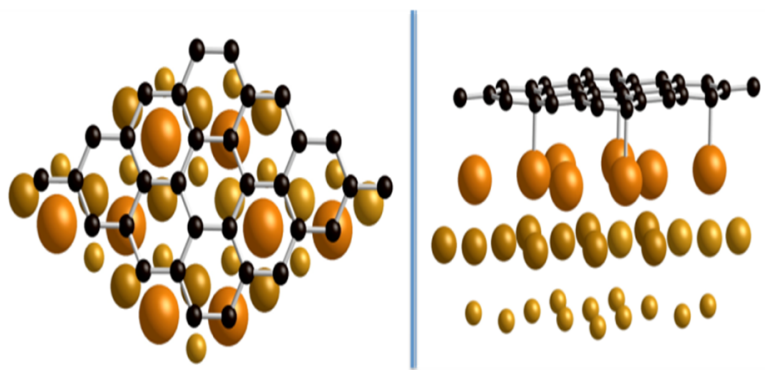

FIG. 2. (Color online) Top view (left) and side view (right) of the structural model derived from DFT calculations for the $\sqrt{3} \times \sqrt{3} \mathrm{R} 30^{\circ}$ reconstruction. Smaller sizes on the spheres represent deeper atoms. The side view is slightly tilted for a better visualization of the vacancy channels and interaction between $\mathrm{C}$ and surface $\mathrm{Pt}$ atoms.

$1 \mathrm{hr})$ in ultra high vacuum conditions $\left(7 \times 10^{-10} \mathrm{mbar}\right)$ the equivalent coverage of $3 \mathrm{ML}$ of molecular precursor on a $\mathrm{Pt}(111)$ surface held at $900-1000 \mathrm{~K}$. The substrate temperature is chosen as the minimum temperature for molecular decomposition. We have found that both, low formation temperatures (900-1000 K) and the use of large polycyclic aromatic precursors, such as $\mathrm{C}_{60} \mathrm{H}_{30}[13]$ or $\mathrm{C}_{60}$ fullerenes, instead of the standard ethylene, strongly favors the amount of the $\mathrm{R} 30^{\circ}$ areas with respect to Moires structures. See EPAPS Document No. [X] for more experimental details. Using these preparation conditions large STM images show that about $15-20 \%$ of the sample is covered with islands exhibiting this reconstruction. Although the relative ratio of the $\mathrm{R} 30^{\circ}$ areas with respect to Moire structures change upon experimental conditions, the kind of structures formed are always the same. LEED patterns and the attenuation of the XPS signal suggest that a single carbon layer remains on the surface, in good agreement with previous studies.[6] STM images were acquired both at room temperature and at $70 \mathrm{~K}$, and the use of different tunneling parameters show in all cases images with similar atomic features.

The R $30^{\circ}$ structure appears in large areas sometimes covering the whole terrace length (up to $100 \mathrm{~nm}$ ). Fig. 1a corresponds to a wide-scan topographic STM image obtained on submonolayer graphene coverage where clean $\mathrm{Pt}$ terraces coexist with Moires and R30 ${ }^{\circ}$ regions. Every one of these regions have been identified by high magnification images on the labeled areas. On Moire regions we observe nanobubbles recently reported [9]. Therefore, each domain shows long-range order convincingly. The R30 phase coexists with Moire regions of different periodicities in the form of crystalline islands (see Fig. 1b), and it has never been found isolated[7, 8]. Fig. 1c shows a detailed STM image where the atomic features forming the $\mathrm{R} 30^{\circ}$ reconstruction are clearly identified. These images are characterized by bright points and depressions aligned along the $[11 \overline{2}]$ surface crystallographic direction indicated in the figure by an arrow. This structure ex-

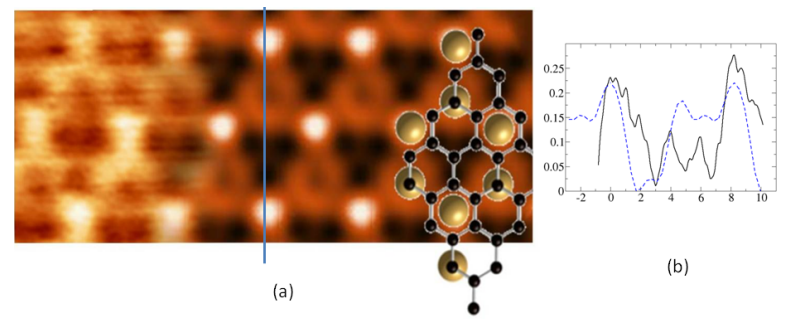

FIG. 3. (Color online) (a) STM images $(\mathrm{V}=0.1 \mathrm{~V})$ for the $\sqrt{3} \times \sqrt{3} \mathrm{R} 30^{\circ}$ experimental (left) and DFT best fit simulation (right). A schematic atomic model has been overlaid. The green horizontal line indicates the merging area between both images, and the blue one is related to the experimental (continous) and theoretical (dashed) scan lines on (b).).

hibits STM images with large atomic corrugation, ranging from 0.60 to $0.15 \AA$, about 5 times higher than those we obtained (already reported by others) for the corresponding Moire regions in the same conditions $(\leq 0.1$ $\AA$ ) [3]. Electronic effects are important in the image appearance and the atomic assignment of STM features in Fig. 1c requires first-principles calculations.

We have used Ab-initio Density Functional Theory (DFT) to locate the equilibrium ground state for the system by searching for a total energy global minimum. Wavefunctions are expanded in plane-waves up to a cutoff of $350 \mathrm{eV}$ and are sampled on a Monkhorst-Pack $6 \times 6 \times 1$ mesh inside the Brillouin zone. $\mathrm{C}$ and $\mathrm{Pt}$ atoms are described by ultra-soft pseudopotentials and the local density approximation is used for the exchange and correlation potential[14]. Actual calculations are performed with the CASTEP code[15]. This formalism yields lattice parameters for bulk fcc platinum of $2.774 \AA$, (fractional error $-0.05 \%)$ and for an isolated graphene layer of $2.440 \AA$ (fractional error $-0.87 \%$ ). The clean $\operatorname{Pt}(111)$ surface shows an outwards relaxation of $+1.0 \%$ and a second layer contraction of $-0.2 \%$, in good agreement with experimental determinations $(+0.8 \%$ and $0.0 \%)$.

A $30^{\circ}$ rotation between the $\mathrm{Pt}$ and $\mathrm{C}$ structures reduces the mismatch between $\mathrm{Pt}$ and graphene meshes from $11 \%$ to about $3 \%$. This is still large and elastic distortions in the graphene layer make the total energy increase, which can be interpreted as an effective repulsive interaction between graphene and platinum. Under these conditions the graphene layer stays at a relatively away from the outermost $\mathrm{Pt}(111)$ layer $(3.31 \AA)$, remaining atomically flat with very little buckling $(\Delta z=0.01$ $\AA$ ). These values are similar to those calculated for the low-interacting Moire regions[3, 12], and in contrast to large atomic corrugations measured in Fig. 1c.

A different way to release stress is to induce a reconstruction on the $\mathrm{Pt}(111)$ surface. We have found that the formation of an ordered network of Pt vacancies at the outermost layer leads to an energetically stable structure compatible with the STM images. After trying many dif- 


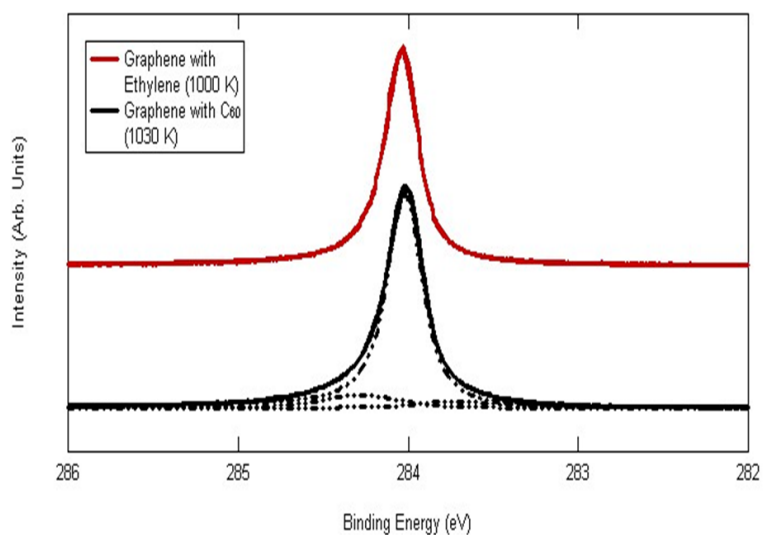

FIG. 4. (Color online) C1s core level spectra of graphene grown on $\mathrm{Pt}(111)$. Upper spectrum: ethylene decomposition (red). Lower spectrum: polycyclic molecular precursors with favourable experimental conditions for the $\mathrm{R} 30^{\circ}$ phase (black). Dashed lines correspond to the best-fit components.

ferent structures (including lateral shifts in the graphene layer, extra carbon atoms, and vacancies in both layer and surface) our best-fit model is depicted in Fig. 2. In this model one surface $\mathrm{Pt}$ atom is missing per $\mathrm{R} 30^{\circ}$ unit cell, and the rest of the Pt atoms are alternatively in top or hollow positions with respect to the graphene layer. DFT calculations show that graphene binds more strongly to the platinum surface in the presence of vacancies. The interaction becomes three times larger $(-0.60$ $\mathrm{eV} /$ per unit cell), and the bond length between the $\mathrm{C}$ near a top Pt shortens to $2.28 \AA$ (Fig. 2, side view). The remaining seven $\mathrm{C}$ atoms in the unit cell relax outwards, producing a maximum buckling in the $\mathrm{C}$ layer of $\Delta z=0.2 \AA$. This number is smaller than the one quoted for long-range ripples in graphene[11]. The outermost $\mathrm{Pt}$ layer goes from expansion to $-8.1 \%$ contraction.

To accurately relate this structural model with the experimental STM images we have performed theoretical simulations using a non-equilibrium Keldysh's Green Functions formalism including multiple scattering events. FIREBALL has been used to compute a DFT Hamiltonian based on a linear combination of orbitals.[16] A tip made with 105 tungsten atoms forming a pyramidal cluster ending in a single atom on a W(100) terrace has been used. STM simulations have been performed on the structural model previously optimized using the more accurate plane-waves formalism. Fig. 3a compares the calculated STM image with a high-resolution experimental image. Experimental and theoretical scan lines along the dotted line are compared in Fig. 3b. Important features in the experiment, i.e., bright spots and depressions surrounded by a tiny halo along the $[11 \overline{2}]$ surface direction are very well reproduced in our simulations, and even a moderate quantitative agreement can be claimed in the scan lines. Total corrugation of the theoretical model for different tip distances range from 0.1 to 0.3
$\AA$, in good agreement with the experimental values. Although C-atoms are $\geq 2 \AA$ above the outermost Pt layer, it is important to notice that the STM image essentially reflects the Pt-atoms because of the large difference in the DOS contribution around the Fermi Level. Additionally, and differently to the Moire regions, imaging of the $\mathrm{C}$ atoms in the $\mathrm{R} 30^{\circ}$ superstructure is prevented because the tip-surface distance has been reduced more than $1 \AA$, increasing the Pt-tip hopping effect by a factor 16.[16] Finally, the presence of the $\mathrm{C}$ atoms from the graphene layer originates multiple scattering processes in the tunneling current, which are responsible for the tiny circular features observed in the STM images around the $\mathrm{Pt}$ atoms that have a $\mathrm{C}$ on top (Fig. 3a).

This atomic model agrees very well with STM images, and it is also supported by high-resolution X-Ray Photoemission Spectroscopy (XPS) data recorded in the SuperESCA beamline of the synchrotron radiation facility ELETTRA. Fig. 4 shows the $\mathrm{C}_{1 s}$ core level spectra of graphene prepared by ethylene dissociation following the standard recipe described in $\operatorname{refs}[3,4,6]$ (upper spectrum), and the one using $\mathrm{C}_{60}$ as molecular precursors. In the latter case the STM images show coexistence of several phases, including the $\mathrm{R} 30^{\circ}$ reconstruction (lower spectrum). The peak corresponding to the ethylene preparation shows a single component centered at $284.0 \mathrm{eV}$, whereas the lower peak corresponding to graphene grown with $\mathrm{C}_{60}$ shows extra components at both sides of the main peak $(284.3 \mathrm{eV}$ and $283.7 \mathrm{eV})$. Thus, the main peak corresponds to the low-interacting Moire regions, and its binding energy is in agreement with previously published results.[6] The small components can be related to the existence of the covalently bound structures. In [6] it has been suggested that the core level peak of $\mathrm{C}$ atoms interacting with the substrate is shifted towards higher binding energies. In the model proposed in Fig. 2 for the R30 $30^{\circ}$ phase there are three different types of $\mathrm{C}$ atoms: over a $\mathrm{Pt}$ vacancy, on top of a surface $\mathrm{Pt}$ and in bridge position. These three $\mathrm{C}$ atoms present different total charges, as depicted by CASTEP calculations: $-4.08 \mathrm{e},-4.15$ e and -4.08 e, respectively. We expect this charge redistribution to be reflected in the XPS $\mathrm{C}_{1 s}$ core level spectra. Moreover, a XPS calculation of the areas involved in different structures gives a value of about $21 \%$ of the sample covered with these covalently bound phases, in good agreement with the STM observation of $15-20 \%$ for this experimental conditions.

In this structural model graphene forms new bonds with $\mathrm{Pt}$ atoms as seen by analyzing the charge density difference for the optimum configuration displayed in Fig. 2: $\mathrm{C} p_{z}$ orbitals interact with $\mathrm{Pt} d_{z^{2}}$ ones accumulating charge in the intermediate region. The main effect of vacancies on the $\mathrm{Pt}$ electronic structure is a subtle one: the d-band narrows and moves down in energy towards the Fermi energy by about $0.3 \mathrm{eV}$. Therefore, the center of mass for the $\mathrm{Pt}$ d-band gets closer to the $\mathrm{C} \pi$-band, 
increasing hybridization and chemical interaction. From the point of view of the electronic structure of carbon atoms the main change is an important increase in the density of states at the Fermi level for $\pi$ bands, which could enhance catalytic properties locally.

The proposed $\mathrm{Pt}$ surface reconstruction might be thought unlikely since it involves kinetic barriers larger than $3 \mathrm{eV}$. However, the existence of vacancies after deposition and decomposition of large organic molecules is widely supported by different experimental observations. First, it has been shown that a graphene layer interacting with the substrate via weak van-der Waals forces tends to form ripples with large spatial periodicities[17]. However, the $\mathrm{R} 30^{\circ}$ regions shown in Fig. 1 do not show ripples, but an atomic corrugation $\geq 5$ than in the Moire regions. This suggests that covalent bonds have been formed between the layer and the substrate. Second, STM images for clean Pt(111) show typical large terraces separated by steps running parallel to the surface crystallographic directions. STM experiments for graphene on $\operatorname{Ru}(0001)$ and $\operatorname{Ir}(111)$ show that steps remain sharp and adjusted to surface crystallographic directions after the graphene layer has been created. In our case, however, when the $\mathrm{R} 30^{\circ}$ phase is on the surface, we observe a rounding of the surface steps, as can be seen in Fig. 1 for an incomplete coverage. See EPAPS Document No. $[\mathrm{X}]$ for more STM images showing this effect. The step smoothing process has been observed for different graphene overlayers. For instance, in the case of the $\sqrt{19} \times \sqrt{19} \mathrm{R} 23.4^{\circ}$ formed after decomposition of benzene at high temperature, a reorganization of the last $\mathrm{Pt}$ layer has been suggested. [8] Rounding of steps is usually associated to transport of $\mathrm{Pt}$ atoms from the terraces, and we introduce the following model to explain this experimental evidence: upon annealing the molecular precursors, a Pt atom per $\mathrm{R} 30^{\circ}$ unit cell is removed from the surface and diffuses towards the step edges, leaving a vacancy behind. [8, 18] The energy necessary to overcome the large kinetic barrier should be found in the complex reorganization of the large carbon-based molecules used to grow the graphene layer. Indeed, it has been reported in the literature that annealing at lower temperatures of different organic molecules, like fullerenes, results in the ejection of $\mathrm{Pt}$ atoms out of the surface, creating vacancies on the $\mathrm{Pt}$ surface layer.[18] The same mechanism has been proposed for fullerenes on $\operatorname{Pd}(110)[19]$, and more recently for $\operatorname{Ag}(111)[20]$ and $\operatorname{Pt}(110)[21]$. In these cases, the $\mathrm{C}_{60}$ adsorb with a hexagon on the vacancy maximizing the number of C-Pt bonds. However, in our case, the orientation of the $\mathrm{C}$ layer with respect to the substrate is imposed by minimization of the stress between both structures resulting in a $\mathrm{C}$ atom placed on top of the vacancy. Furthermore, the clean $\mathrm{Pt}(111)$ surface is known to show a tendency to reconstruct at high temperatures.[22] The organic polycyclic molecules used as precursors get enough energy to facilitate the breaking of strong $\mathrm{C}-\mathrm{C}$ bonds and decompose once in contact with the reactive surface $\mathrm{Pt}$ atoms. The adsorption, bondbreaking and interaction of carbon species modifies the energetic balance resulting in the formation of new binding forces, that induce the formation of vacancies. Thus we conclude that the mechanism of vacancy formation in the platinum (or Pd) surfaces upon annealing is likely a general one in the interaction of large carbonaceous species with Pt crystals.

We acknowledge financial support from MAT20081497 and CSD2007-41 (Spain), and the 7th Framework Program (FP7/2007-2013) (EU). C.G. acknowledge CSIC "JAE-Doc" and P.M. INTA "Rafael Calvo Rodes".

[1] K. S. Novoselov, A. K. Geim, S. V. Morozov et al., Science 306, 666 (2004).

[2] F. Schedin, A. K. Geim, S. V. Morozov et al., Nature 6, 652 (2007).

[3] A. L. V. de Parga, F. Calleja, B. Borca et al., Phys. Rev. Lett. 100, 056807 (2008).

[4] W. Moritz, B. Wang, M. L. Bocquet et al., Phys. Rev. Lett. 104, 136102 (2010).

[5] A. T. N.'Diaye, J. Coraux, T. N. Plasa et al., New Journal of Physics 10, 043033 (2008).

[6] A. B. Preobrajenski, M. L. Ng, A. S. Vinogradov, and N. Martesson, Phys. Rev. B 78, 073401 (2008).

[7] T. A. Land, T. Michely, R. J. Behm et al., Surf. Sci. 264, 261 (1992).

[8] T. Fujita, W. Kobayashi, and C. Oshima, Surf. Interface Anal. 37, 120 (2005).

[9] N. Levy, S. A. Burke, K. L. Meaker et al., Science 329, $544(2010)$.

[10] J. Lahiri, Y. Lin, P. Bozkurt et al., Nature Nanotechnology 5, 326 (2010).

[11] B. Wang, S. Gunther, J. Wintterlin, and M. L. Boquet, New Journal of Physics 12, 043041 (2010).

[12] G. Giovanneti, P. A. Khomayakov, G. Brocks et al., Phys. Rev. Lett. 101, 026803 (2008).

[13] G. Otero, G. B. an C. Sanchez-Sanchez et al., Nature 454, 865 (2008).

[14] D. M. Ceperley and B. J. Alder, Phys. Rev. Lett. 45, 566 (1980).

[15] S. Clark, M. D. Segall, C. Pickard et al., Z. fuer Kristallographie 220, 567 (2005).

[16] J. M. Blanco, F. Flores, and R. Perez, Prog. Surf. Sci. 81, 403 (2006).

[17] W. Bao, F. Miao, Z. Chen et al., Nature Nanotechnology 4, 562 (2009).

[18] R. Felici, M. Pedio, F. Borgatti et al., Nature Materials 4, 688 (2005).

[19] J. Weckesser, J. V. Barth, and K. Kern, Phys. Rev. B 64, 161403 (2001).

[20] H. Li, K. Pussi, K. J. Hanna et al., Phys. Rev. Lett. 103, 056101 (2009).

[21] X. Torrelles, V. Langlais, M. D. Santis et al., Phys. Rev. B 81, 041404 (2010).

[22] A. R. Sandy, S. G. J. Mochrie, D. M. Zehner et al., Phys. Rev. Lett. 68, 2192 (1992). 